\title{
FATORES DE RISCO E PROTEÇÃO PARA O SUICÍDIO NA ADOLESCÊNCIA: uma revisão de literatura
}

\author{
Alessandra Soares Cardoso \\ Alessandra Marques Cecconello ${ }^{2}$
}

Resumo: Este estudo pretende analisar os fatores de risco e proteção relacionados ao suicídio na adolescência. A revisão de literatura utilizou as bases de dados da Biblioteca Virtual da Saúde, Biblioteca Eletrônica Científica Online (Scielo) e Lilacs, envolvendo artigos dos últimos dez anos (2009-2019), no idioma português. Dentre os 186 artigos encontrados, foram utilizados 18 artigos, selecionados a partir dos critérios para inclusão neste estudo.A adolescência é uma fase na qual ocorrem diversas mudanças, entre alterações físicas e psicológicas. Nesta passagem do ciclo vital, o adolescente está redescobrindo sua sexualidade e se reafirmando como jovem, desenvolvendo novos vínculos sociais, estando, assim, mais suscetível para se expor a comportamentos de risco. Destaca-se aqui neste estudo os fatores de risco relacionados ao suicídio na adolescência, dentre eles, características de personalidade, transtornos mentais, doenças físicas orgânicas, estressores familiares, violência intrafamiliar, estressores escolares, comportamentos de risco, estressores psicossociais, fatores psicológicos e estressores sócio econômicos. Dentre os fatores de proteção, destaca-se características individuais, apoio familiar e apoio social.Os resultados deste trabalho indicam a importância de fortalecer o papel da família e da escola, principalmente em relação ao estabelecimento de vínculos com os adolescentes, pois observa-se que o contexto familiar e escolar podem atuar como risco ou proteção, dependendo da qualidade das relações estabelecidas nestes ambientes.

Palavras-Chaves: Suicídio. Adolescência. Fatores de Risco e Fatores de Proteção.

\footnotetext{
${ }^{1}$ Acadêmica do Curso de Bacharelado em Psicologia da UNICNEC.

${ }^{2}$ Doutora em Psicologia do Desenvolvimento pela Universidade Federal do Rio Grande do Sul (UFRGS). 
RISK FACTORS AND PROTECTION FOR SUICIDE IN ADOLESCENT: a literature

review

\begin{abstract}
This study aims to analyze risk and protection factors related to suicide in adolescence. The literature review used the databases of the Virtual Health Library, Online Scientific Electronic Library (Scielo) and Lilacs, involving articles from the last ten years (2009-2019), in Portuguese. Among the 186 articles found, 18 articles were selected, using the criteria for inclusion in this study. Adolescence is a phase in which several changes occur, between physical and psychological changes. In this passage of the life cycle, adolescents are rediscovering their sexuality and reaffirming themselves as young people, developing new social bonds, thus being more susceptible to risky behaviors. Here we highlight the risk factors related to suicide in adolescence, including personality characteristics, mental disorders, organic physical illnesses, family stressors, intrafamily violence, school stressors, risk behaviors, psychosocial stressors, psychological factors and socio-economic vulnerability. Among the protective factors, individual characteristics, family support and social support stand out. The results of this work indicate the importance of strengthening the role of family and school, especially in relation to the establishment of bonds with adolescents, since it is observed that the family and school context can act as risk or protection, depending on the quality of these relationships. established in the environment.
\end{abstract}

Keywords: Suicide. Adolescence. Risk factors and protection factors

\title{
1 INTRODUÇÃO
}

A adolescência é compreendida como um processo transitório importante do desenvolvimento humano entre a fase da infância e a fase adulta. Segundo a Organização Mundial da Saúde(2005) a adolescência configura-se no período entre 10 e 19 anos. No Brasil, segundo o Estatuto da Criança e do Adolescente, essa faixa etária é compreendida entre 12 e 18 anos (BARROS; PICHELLI; RIBEIRO, 2016).

A adolescência é entendida como uma fase de "muitas turbulências", mudanças fisiológicas e psicológicas, além das questões afetivas, familiares e sociais. Neste sentido, o adolescente se torna mais vulnerável a influências. Além de estar desenvolvendo sua 
autonomia, o jovem adolescente preocupa-se com seu papel na sociedade, projetando seus sonhos, planos, hobbies, estudos, profissão, grupos sociais e a construção de sua identidade (BARROS; PICHELLI; RIBEIRO, 2016).

Nesse processo de construção da identidade da saída da infância e entrada na vida adulta, a transição do adolescente por vários contextos pode levar ao aparecimento de comportamentos de risco, como abuso de substâncias psicoativas, comportamento sexual de risco e violência (NIQUICE, 2014). De acordo com a OMS, existem três tipos de violência: a coletiva, a interpessoal e a autoinfligida. Este estudo abordou a violência autoinfligida.

A violência autoinfligida é definida por toda a ação praticada pela própria pessoa que cause dano a sua saúde, colocando sua vida em risco (CAMARGO, et al, 2011). O comportamento suicida é entendido como uma manifestação da violência autoinfligida.

O comportamento suicida consiste em qualquer ato intencional de causar dano a si mesmo com o objetivo final acabar com sua própria vida (SCHLOSSER; ROSA; MORE, 2014).Segundo Magnani e Staudt(2018), o comportamento suicida pode ser dividido em três categorias: ideação, tentativa e suicídio consumado. A ideação refere-se a pensamentos para pôr fim à própria vida. A tentativa é configurada pelo comportamento autodestruidor o qual não se concretiza no ato final. Importante estar atento a esses comportamentos, tendo em vista que uma vez realizada a tentativa, a tendência é repetir esse comportamento. O suicídio é considerado como o comportamento autodestrutivo consumado.

O suicídio é entendido como um problema muito grave de saúde pública. Configura-se entre as dez importantes causas de morte no mundo, abrange o terceiro lugar entre os indivíduos de 15 a 34 anos (BAGGIO,2009). Assim, observa-se que os adolescentes se encontram nesta faixa etária de risco para o suicídio, torna-se muito importante refletir sobre os fatores que podem contribuir para isso.

Nesta fase de grandes mudanças, o jovem está mais vulnerável, pois muitas vezes tem dificuldades de lidar com situações estressantes e emoções. Na adolescência, o jovem lida de uma forma mais intensa com suas emoções, estando mais propenso a comportamentos impulsivos podendo se tornar um fator de risco para o comportamento suicida(MAGNANI; STAUDT, 2018).

No entanto, nem sempre a adolescência vai ser palco de comportamentos de risco, pois depende da interação com outros fatores, como os fatores de proteção. Uma adolescência patológica, entretanto, é marcada pela presença de fatores de risco. Entre os fatores de risco 
para o comportamento suicida na adolescência, pode-se destacar a negligência da família em atender as necessidades de cuidado e afeto dos filhos, a perda de pessoas próximas da família, a exposição à violência física, psicológica e sexual, condições de saúde desfavoráveis, o isolamento social, o uso de substâncias químicas, sentimentos de solidão, bullying, baixo rendimento escolar, baixa autoestima, dinâmica familiar conflituosa, entre outros (BRAGA; DELL'AGLIO, 2013). A presença de fatores de risco na adolescência pode contribuir para a vivência de uma adolescência patológica, aumentando, consequentemente, o risco para o comportamento suicida.

O suicídio na adolescência ainda é visto como um tabu. No entanto, se faz importante e necessário refletir sobre este fato, cujos índices vêm aumentando consideravelmente ao longo dos anos. O objetivo desta pesquisa é identificar os fatores de risco para o suicídio na adolescência, bem como analisar os fatores de proteção para a prevenção deste grave problema de saúde.

\section{METODOLOGIA}

Este trabalho foi desenvolvido a partir de uma revisão de literatura, definida por Koller, Paula e Hohendorff (2014) como uma pesquisa científica na qual se pretende analisar dados de um determinado tema definido. A revisão utilizou as bases de dados da Biblioteca Virtual da Saúde, Biblioteca Eletrônica Científica (Scielo) e Lilacs.

Foram realizadas as buscas em agosto de 2019, envolvendo artigos dos últimos dez anos (2009-2019), no idioma português. As palavras-chaves utilizadas foram "adolescência", "suicídio", "fatores de risco", "fatores de proteção". Foram critérios de exclusão artigos publicados antes de 2009. As buscas nas bases de dados foram realizadas apartir de três combinações: (1) adolescência e suicídio; (2) fatores de risco e suicídio na adolescência; (3) fatores de proteção e suicídio na adolescência. Dos 186 artigos encontrados, foram utilizados 18 artigos. Após a leitura dos resumos dos artigos, alguns se repetiram e outros não preencheram os critérios, sendo, então, excluídos 6 artigos.

Tabela 1 - Artigos encontrados nas bases de dados

\begin{tabular}{|c|c|c|c|}
\hline $\begin{array}{c}\text { Base de dados } \\
\text { Total: } 186\end{array}$ & Scielo & BVS & Lilacs \\
\hline Total & 80 & 96 & 10 \\
\hline
\end{tabular}




\begin{tabular}{|c|c|c|c|}
\hline Aceitos & 5 & 12 & 1 \\
\hline Descartados & 5 & 4 & 2 \\
\hline
\end{tabular}

Fonte: Elaborado pela autora, 2019.

\section{RESULTADOS E DISCUSSÃO}

Os fatores de risco e proteção para o suicídio na adolescência foram analisados, categorizados e organizados em tabelas. Serão apresentadas a seguir as Tabelas 2 e 3, com os Fatores de Risco e os Fatores de Proteção para o suicídio na adolescente, seguidas respectivamente da sua discussão.

Tabela 2 - Fatores de Risco para o Suicídio Adolescente.

\begin{tabular}{|c|c|}
\hline 1 Características de Personalidade & $\begin{array}{l}\text { - } \quad \text { Temperamento; } \\
\text { - } \quad \text { Traços de impulsividade e agressividade, timidez; } \\
\text { - } \quad \text { Baixa autoestima; }\end{array}$ \\
\hline 2 Transtornos Mentais & $\begin{array}{l}\text { - Transtornos de personalidade (Bordeline, narcisista, anti } \\
\text { social); } \\
\text { - } \quad \text { Transtornos de humor (Bipolar e Depressão); } \\
\text { - } \quad \text { Transtornos decorrentes do uso de substâncias psicoativas; } \\
\text { - } \quad \text { Transtornos psicóticos (esquizofrenia); } \\
\text { - Transtornos de ansiedade; } \\
\text { - } \quad \text { Transtornos de conduta (transtorno opositor desafiante); }\end{array}$ \\
\hline 3 Doenças Físicas Orgânicas & $\begin{array}{l}\text { - Doenças associadas a diabetes e epilepsia; } \\
\text { - Doenças crônicas incapacitantes; }\end{array}$ \\
\hline 4 Estressores Familiares & $\begin{array}{l}\text { - } \quad \text { Fragilidade dos vínculos parentais; } \\
\text { - } \quad \text { Práticas parentais inadequadas, Alcoolismo dos pais; } \\
\text { - Uso de drogas pelos pais; } \\
\text { - } \quad \text { Transtorno mental dos pais; } \\
\text { - Histórico de suicídio na família; }\end{array}$ \\
\hline 5 Violência Intrafamiliar & $\begin{array}{ll}\text { - } & \text { Abuso físico; } \\
\text { - } & \text { Abuso emocional; } \\
\text { - } & \text { Abuso sexual; } \\
\text { - } & \text { Negligência; } \\
\text { - } & \text { Violência conjugal; }\end{array}$ \\
\hline
\end{tabular}




\begin{tabular}{|c|c|}
\hline 6 Estressores Escolares & $\begin{array}{l}\text { - } \quad \text { Bullyng; } \\
\text { - } \quad \text { Rejeição; } \\
\text { - } \quad \text { Exclusão social; } \\
\text { - } \quad \text { Amizades com jovens problemáticos; } \\
\text { - } \quad \text { Baixo rendimento escolar; } \\
\text { - } \quad \text { Falta de comprometimento com os estudos; } \\
\text { - } \quad \text { Baixa escolaridade; }\end{array}$ \\
\hline 7 Comportamentos de Riscos & $\begin{array}{l}\text { - Condutas sexuais de risco (HIV, Dst's, gravidez, relações } \\
\text { sexuais desprotegidas); } \\
\text { - } \quad \text { Usode drogas e álcool; } \\
\text { - } \quad \text { Delinqüência e criminalidade; }\end{array}$ \\
\hline 8 Estressores Psicossociais & $\begin{array}{ll}\text { - } & \text { Perdas, } \\
\text { - } & \text { Desilusão amorosa e rompimento amoroso. }\end{array}$ \\
\hline
\end{tabular}

(continuação)

Tabela 2 - Fatores de Risco para o Suicídio Adolescente.

\begin{tabular}{|c|c|}
\hline 9 Fatores Psicológicos (Sentimentos) & $\begin{array}{l}\text { - Sentimentos de solidão, } \\
\text { - Desesperança, } \\
\text { - Desamparo e não pertencimento, sentimentos de culpa e } \\
\text { inferioridade, } \\
\text { - Sentimentos negativos, } \\
\text { - Sentimentos de abandono e negligência, necessidade de se } \\
\text { sentir aceito. }\end{array}$ \\
\hline 10 Estressores Sócio Econômicos & $\begin{array}{ll}\text { - } & \text { Pobreza, } \\
\text { - } & \text { Baixo nível socioeconômico }\end{array}$ \\
\hline
\end{tabular}

Fonte: Elaborado pela autora, 2019

A tabela 2 apresenta vários fatores de risco para o comportamento suicida na adolescência. Entre eles, é possível destacar: características de personalidade, transtornos mentais, doenças físicas e orgânicas, estressores familiares, violência intrafamiliar, estressores escolares, comportamentos de risco, estressores psicossociais, fatores psicológicos e estressores sócio econômicos.

As características de personalidade são identificadas como um importante fator de risco para o suicídio, pois como afirma Sá, et al (2010), os traços de impulsividade e medo são constituídos ao longo da formação da criança em seu processo de identidade. É importante salientar que um ambiente negligenciador, com violência física, contribui para a formação de traços mais agressivos, prejudicando os estímulos afetivos adequados. 
Magnani e Staudt (2018) enfatizam que desenvolvimento da baixa autoestima é apontado como uma forte característica de risco relacionada ao comportamento suicida na adolescência. A falta de apoio e supervisão dos pais pode favorecer o jovem a desenvolver baixa autoestima, tendo em vista que nesta fase o jovem precisa de afeto, apoio e a presença dos pais.

Os transtornos mentais são apontados como fatores de risco para o comportamento suicida, tendo em vista que, na adolescência, esses fatores podem ser potencializados em função das mudanças do ciclo vital e com o ambiente familiar em que este jovem está inserido (MAGNANI; STAUDT,2018).

Diversos autores afirmam que a depressão, os transtornos psicóticos, os transtornos de ansiedade, os transtornos de conduta, os transtornos alimentares eos transtornos de estresse pós-traumático estão relacionados ao comportamento suicida, tendo em vista que o jovem em depressão possui uma visão mais negativa do mundo, aumentando a probabilidade de se expor em comportamentos de risco (BARROS; PICHELLI; RIBEIRO, 2016; SCHLOSSER; ROSA; MORE, 2014).

Dentre as doenças físicas e orgânicas, é possível ressaltar HIV/AIDS, epilepsia, trauma medular e neoplasias malignas, que são apontados na literatura como fatores de risco para o comportamento suicida. O alto índice de sofrimento emocional dos adolescentes parece estar relacionado também à dificuldade de lidar com os problemas e compreender o significado da vida e da morte (CERQUEIRA; LIMA, 2015;ARAÚJO; VIEIRA; COUTINHO,2010).

A literatura aponta vários estudos relacionados com as fragilidades dos vínculos parentais. Pais que não possuem uma relação harmoniosa e de afetividade com seus filhos podem contribuir para o comportamento suicida. Práticas parentais inadequadas também contribuem para isto: dentre as práticas observadas nesta revisão, destacamos que pais permissivos, negligentes, autoritários e indulgentes são citados como estilos parentais inadequados (ZAPPE; DAPPER,2017).

Da mesma forma, a instabilidade familiar e falta de afeto dos pais são apontados como importantes fatores de risco, ocasionando negligência e falta de cuidados emocionais, expondo o adolescente a comportamentos de risco (SILVA,et al, 2015).

Couto e Tavares (2016), em suas pesquisas, relacionam a influência do apego com a saúde mental dos adolescentes. Relatam que adolescentes com apego inseguro possuem uma Perspectiva: Ciência e Saúde,Osório, V. 4 (2): 101-117, Dez 2019. 
maior tendência a apresentarem sintomas de depressão e ansiedade, uma maior chance de desenvolverem transtornos psicológicos.

O alcoolismo dos pais e o uso de drogas pelos mesmos são comportamentos de risco que podem ser seguidos pelos adolescentes, tendo em vista a tendência à repetição de padrões de comportamentos transgeracionalmente pelos filhos (SÁ,et al,2010).

Em relação aos estressores escolares, pesquisas realizadas por Moreira e Bastos (2009) apontam que o bullying e a vitimização estão presentes em diversos contextos escolares, causando prejuízo social e cognitivo aos adolescentes. Vilhena e Paula(2017) apontam que sentimentos de rejeição, vitimização e baixo rendimento escolar são fatores de risco na adolescência. Importante salientar que, muitas vezes, essas práticas de rejeição começam na família, possuindo um papel fundamental na vida do jovem adolescente, e são acentuadas na vida escolar.

Sá, et al(2010) apontam que o baixo rendimento escolar pode estar relacionado com o desinteresse dos pais frente às atividades desenvolvidas pelos filhos. Importante destacar que esse desinteresse afeta diretamente o comportamento adolescente, podendo contribuir para comportamentos de risco.Zappe e Dapper (2017) observaram que adolescentes podem ser influenciados pelos grupos sociais a praticarem atos que se coloquem em situações de risco, aumentando a vulnerabilidade para influências negativas em seu desenvolvimento.

Nas pesquisas de Couto e Tavares (2016), a violência intrafamiliar é pontuada como um importante fator de risco para os adolescentes. Adolescentes expostos a abuso sexual na infância tiveram maiores índices de tentativas de suicídio.

Barros, Pichelli e Ribeiro (2016)afirmam que, dentre as diversas formas de violência, a violência intrafamiliar se destaca como extremamente danosa, pois o primeiro ambiente no qual o adolescente irá se socializar é a família, tendo um papel fundamental neste processo.Schlosser, Rosa e More (2014) afirmam que maus tratos e negligência estão presentes em diversos contextos familiares e são considerados fatores de risco para o adolescente, tendo em vista que um ambiente pobre de afeto e com dificuldades em satisfazer as necessidades emocionais dos filhos desencadeia sofrimento psíquico, expondo o adolescente a comportamentos de risco.

Vilhena e Paula (2017) relatam que violência entre o casal e nas relações entre os filhos, assim como práticas agressivas e negligentes, afetam o funcionamento psicológico do 
adolescente. A violência física, a violência psicológica e a sexual são pontuadas como importantes fatores de risco para o adolescente. (PATIAS; SILVA; DELL'AGLIO,2016)

Patias, Silva e Dell'aglio (2016)afirmam que condutas sexuais de risco e uso de drogas e álcool aumentam a vulnerabilidade do adolescente.Azevedo e Dutra (2012) enfatizam que a gravidez na adolescência também expõe o adolescente a situações de risco, tendo em vista que traz ao adolescente uma responsabilidade para a qual ele não está ainda preparado psicologicamente. A gravidez na adolescência seria entendida como uma forma de estabelecer uma relação com o parceiro.

Vilhena e Paula (2017) relacionaram o início precoce do comportamento sexual em adolescentes como um importante fator de risco. Enfatizaram também que a delinquência e a criminalidade estão presentes em adolescentes vulneráveis.Moreira e Bastos (2009)verificaram que o uso de substâncias psicoativas induz os adolescentes a se colocarem em situações de risco, pois estes não conseguem buscar solução para seus problemas,buscando nas drogas uma solução para os mesmos.

A desilusão amorosa e/ou rompimento de uma relação amorosa podem prejudicar o funcionamento psicológico do adolescente, tendo em vista que, na adolescência, iniciam-se os primeiros relacionamentos amorosos e o jovem ainda não tem a maturidade para lidar com certas frustrações (AZEVEDO; DUTRA, 2012).

Perdas significativas e parentais e a separação dos pais também são fortes indicadores ao suicídio adolescente. Nestes casos, a falta de diálogo e o afastamento de uma das figuras parentais causam uma desestruturação no contexto familiar, marcando significativamente a vida do adolescente (AZEVEDO; DUTRA, 2012).

Pessoas com histórico de suicídio na família possuem uma maior probabilidade de cometerem esse ato devido ao grande sofrimento já ocorrido, levando o indivíduo a pensar no suicídio como uma solução já escolhida por outros familiares(ARAUJO; VIEIRA; COUTINHO, 2010).

Fatores psicológicos relacionados a sentimentos de solidão, desesperança, impotência, culpa, sentir-se sem valor ou com vergonha são apontados como fatores de risco na adolescência (CERQUEIRA; LIMA,2015). Magnani e Staudt(2018) também apontam que adolescentes com dificuldades emocionais, sentimentos de solidão e de não pertencimento possuem uma maior prevalência a comportamentos de risco. Importante salientar que, na adolescência, estes fatores tendem a ser intensificados quando a família não dá o apoio 
emocional necessário ao adolescente, levando o adolescente a se colocar em situações de risco.

Baggio, Palazzo e Aerts (2009) consideram que sentimentos de tristeza e solidão podem estar associados a comportamentos suicidas, bem como sentimentos de abandono e descaso, apontados como grandes geradores a comportamentos de risco. Sentimentos de culpa e vergonha são pontuados como fatores de risco, sendo que o jovem tem dificuldade de expressar seu sofrimento referente a um conflito interno, assim visualizando a morte como uma solução para esse sofrimento. (ARAUJO; VIEIRA; COUTINHO, 2010).

Araújo, Vieira e Coutinho(2010) afirmam que condições econômicas extremas, ou muito ricos, ou muito pobres, podem expor mais os adolescentes a fatores de risco ao suicídio. Vilhena e Paula(2017) consideram que os adolescentes são influenciados pelo meio em que vivem, principalmente em situações de pobreza, onde a exposição a fatores de riscos é maior.Pesquisas realizadas por Silva, et al (2015) apontam que a maioria dos dados colhidos em pesquisas de unidades de urgências públicas verificam a questão da classe econômica mais baixa, porém, este autor salienta que, no caso de classes altas, esse comportamento de risco não chega nas redes públicas e sim redes particulares, necessitando assim de mais pesquisas nesta área particular também.

Como é possível observar, são vários os fatores de risco que podem levar ao suicídio adolescente. A partir de agora, serão analisados os fatores de proteção. A Tabela 3, abaixo, apresenta os fatores de proteção para o suicídio adolescente.

Tabela 3 - Categorias dos Fatores de Proteção ao Suicídio Adolescente.

\begin{tabular}{|l|l|}
\hline 1 Características Individuais & $\bullet$ Habilidades Sociais \\
& $\bullet$ Autoestima \\
\hline 2 Apoio Familiar & $\bullet$ Espiritualidade \\
& $\bullet$ Práticas Parentais adequadas(limites, \\
& \\
\hline 3 Apoio Social & $\bullet$ Escola \\
& $\bullet$ Amigos \\
\hline
\end{tabular}

Fonte: Elaborado pela autora, 2019

Entre os fatores de proteção para o suicídio adolescente, pode-se destacar as características individuais, o apoio familiar e o apoio social. 
Ao contrário dos fatores de risco, os fatores de proteção dizem respeito a condições favoráveis em que a criança/adolescente é exposta, reduzindo desfechos desfavoráveis e aumentando resultados positivos na trajetória comportamental, psicológica e acadêmica desses indivíduos (VILHENA; PAULA, 2017, pag8; apud et al SANT'ANNA, 2011).

Moreira e Bastos(20009)abordam que possuir habilidades sociais, ter confiança em si mesmo, ser uma pessoa receptiva e estar aberta a conselhos são importantes fatores de proteção na adolescência. Da mesma forma, alguns autores apontam que a autoestima e a autoeficácia, a aceitação dos pais e o envolvimento dos mesmos na vida do jovem são considerados fatores de proteção, prevenindo a possibilidade de envolvimento em comportamentos de risco (MAGNANI; STAUDT. 2018; ZAPPE; DELL'AGLIO, 2016).

Patias, Silva e Dell'aglio (2016) afirmam que adolescentes com características e habilidades que favorecem o desenvolvimento da resiliência, tais como esperança, estratégias de coping, autoestima e bem-estar, possuem uma menor tendência e se expor a violência e comportamentos de risco. Vilhena e Paula (2017) salientam que jovens com habilidades sociais possuem um maior desempenho em suas funções escolares, assim como a religiosidade também tem a tendência a ser um fator protetor nesta fase do ciclo vital.Jahn e Dell'aglio(2017) apontam que a espiritualidade ou religiosidade podem promover no adolescente fatores positivos, facilitando, assim, comportamentos mais saudáveis, evitando comportamentos de risco como, consumo de drogas, álcool e fumo, promovendo mais bem-estar e sentimentos de esperança em suas vivências.

Diversos autores destacam que a família tem um papel muito importante na vida do jovem, sendo que as relações com vínculos seguros e afetividade são importantes fatores de proteção (ZAPPE; DELL'AGLIO, 2016; MAGNANI;STAUDT, 2018). As primeiras relações de vínculos seguros favorecem o desenvolvimento de capacidades e habilidades sociais saudáveis no jovem (MAGNANI; STAUDT, 2018). Couto e Tavares(2016) apontam que o apego seguro é um fator de proteção importante na adolescência, jovens com apego seguro têm habilidades de desenvolver autonomia, fazer vínculos de amizades e aumentar sua rede de apoio social. Vilhena e Paula (2017) pontuam que o apego auxilia o jovem a adquirir confiança e segurança em si mesmo, para uma melhor resposta frente ao problema.

Habilidades de comunicação, possibilidades para adquirir novos conhecimentos e habilidade de pedir ajuda são fatores de proteção na adolescência. Bom relacionamento familiar, apoio familiar, figuras parentais dedicadas, que sabem impor os limites e regras de 
uma forma adequada, são fatores de protetivos na adolescência. Da mesma forma, fatores como alimentação saudável, sono, atividade física, ambiente livre de drogas e fumo são pontuados como fatores de proteção relevantes(CERQUEIRA; LIMA, 2015).

Práticas parentais mais adequadas são indicadas como fatores de proteção, pais que acompanham o desenvolvimento dos filhos estando presentes e sendo acolhedores, fortalecem a identidade deste adolescente,minimizando o envolvimento em comportamentos de risco, como uso de álcool e drogas. Um relacionamento de afetividade e disciplina estimula o adolescente a se sentir aceito, seguro e valorizado, contribuindo para seu desenvolvimento saudável (ZAPPE; DAPPER, 2017).

Baggio, Palazzo e Aerts(2009), em suas pesquisas, relacionam a escola como um importante fator de proteção para adolescentes. Sendo considerada como o segundo ambiente onde o jovem está inserido, a escola pode ser o local no qual se identificam os problemas iniciais trazidos pelos adolescentes, as primeiras relações e os comportamentos sociais. Importante destacar que o adolescente está em busca da sua autonomia e identidade, ele vai buscar nas relações de amizades algo que o faça se sentir pertencente a um grupo, fator importante para a construção de sua identidade e personalidade.

Portanto como foi possível analisar, verificamos a importância dos fatores de proteção na adolescência, fortalecendo os vínculos familiares diminuindo a exposição a fatores de risco.

\section{CONSIDERAÇÕES FINAIS}

O presente estudo teve como objetivo analisar os principais fatores de risco e proteção relacionados ao suicídio na adolescência. A adolescência é uma fase composta por diversas mudanças e alterações físicas e psicológicas. Frente a estas mudanças, o jovem está em processo de construção de sua identidade, iniciando suas primeiras relações com o contexto social e possibilitando essa inserção através de suas experiências sociais vivenciadas, podendo estar mais suscetível a se expor a comportamentos de risco, prejudicando em seu desenvolvimento social e cognitivo. Destaca-se aqui a importância da família como papel protetivo na relação dos vínculos sociais adequados, de proteção, cuidado e autonomia. 
Estudos importantes na área da psicologia com adolescentes enfatizam uma grande preocupação com esse público em relação a comportamentos de risco que colocam o adolescente em situações que prejudicam seu desenvolvimento físico e psicológico.

Maus tratos na infância e na adolescência são considerados fatores importantes de risco, podendo levar os jovens a buscarem situações que os fragilizam. Considerando que, para um desenvolvimento saudável, é importante o bem estar físico e psicológico na infância, condições de saúde, alimentação, moradia, segurança, vestuário, entre outros, salientamos que o indivíduo é um ser biopsicossocial, e que os modelos de aprendizagem social são padrões aprendidos na infância.

Os fatores de proteção na adolescência são apresentados como fatores que podem mudar e modificar as respostas relacionadas ao desenvolvimento deste jovem, diminuindo as possibilidades do indivíduo se envolver em situações de risco. Dentre os fatores de proteção, destacamos alguns mais importantes: a escola, os amigos, o apoio social, o apoio familiar. A família tem o papel fundamental na construção desse processo de autonomia da criança, podendo ser destacada como um fator de proteção ou de risco. Destacamos aqui a importância das práticas educativas adequadas como: afeto, cuidado, proteção,autonomia, atendimento as necessidades emocionais e físicas da criança, lazer, limites e regras, comunicação assertiva, desenvolvimento da empatia, responsabilidade e senso de justiça. Dentre os fatores de risco abordados neste estudo, destacamos que a família pode constituir risco quando dispõe de ser um ambiente negligenciador de afeto e cuidados para a criança e adolescente. Da mesma forma, o isolamento social, o uso de substâncias psicoativas, o abandono, as perdas familiares, a exposição à violência física e psicológica, traumas decorrentes de abusos físicos e sexuais, transtornos de humor e personalidade, sentimentos de solidão, bullying, condições de saúde desfavoráveis, baixo rendimento escolar, baixa autoestima, dinâmica familiar abusiva e negligenciadora, desestruturas familiares, entre outras, também constituem riscos.

Neste sentido, a partir da análise dos fatores de risco e proteção para o suicídio na adolescência, observa-se que este é um fenômeno muito complexo, e que suas causas são multifatoriais. O suicídio é considerado um problema muito grave em saúde pública, assim, compete que tenhamos uma atenção ainda maior ao público adolescente,fase em que ocorrem muitas mudanças físicas e psicológicas. É importante desenvolver mais programas de prevenção voltados à saúde mental do adolescente, fortalecendo a sua rede de apoio familiar e social, bem como seus recursos pessoais. Contextos acolhedores, tais como a família e a Perspectiva: Ciência e Saúde,Osório, V. 4 (2): 101-117, Dez 2019. 
escola, fortalecem os fatores de proteção, prevenindo os adolescentes de se colocarem em risco a sua saúde física, social e psicológica.

\section{REFERÊNCIAS BIBLIOGRÁFICAS}

ARAÚJO, Luciene da Costa; VIEIRA,Kay Francis Leal; COUTINHO, Maria da Penha de Lima. Ideação suicida na adolescência: um enfoque psicossociológico nocontexto do ensino médio. Universidade Federal da Paraíba, João Pessoa, Brasil, 2010. Disponivel: $<$ http://www.scielo.br/pdf/pusf/v15n1/06.pdf> Acesso: 01/08/19

AZEVEDO, Ana Karina Silva; DUTRA, Elza Maria do Socorro. Relação amorosa e tentativa de suicídio na adolescência: uma Questão De (Des)amor. Universidade Federal do Rio Grande do Norte (UFRN), 2012. Disponivel:

$<$ http://pepsic.bvsalud.org/pdf/rag/v18n1/v18n1a04.pdf $>$. Acesso em: 01/08/19

BARROS, Paula Danielly Queiroz;PICHELLI, Ana AlaydeWerba Saldanha;

RIBEIRO, Karla Carolina Silveira. Associação entre o consumo de drogase a ideação suicida em adolescentes.Universidade Estadual da Paraíba (UEPB), 2016.Disponível: $<$ http://pepsic.bvsalud.org/pdf/mental/v11n21/v11n21a02.pdf $>$ Acesso em: 01/08/19

BRAGA, Luiza de Lima; DELL'AGLIO, Débora Dalbosco. Artigo Suicídio na Adolescência: Fatores de Risco, Depressão e Gênero. Universidade Federal do Rio Grande do Sul. Porto Alegre. RS, 2013. Disponível: <http://pepsic.bvsalud.org /scie lo.php?script=sci_arttext\&pid=S198334822013000100002 $>$ Acesso: 16/03/19

CAMARGO, Fernanda Carolina; et al. Violência Autoinfligidae anos potenciais de vida perdidos em Minas Gerais, Brasil. Universidade Federal do Triangulo Mineiro (UFTM). Minas Gerais, 2011. Disponível: <http://www.scielo.br/pdf/tce/v20 nspe/v20nspea13.pdf> Acesso em: 01/08/19

CERQUEIRA, YohannaShneideider; LIMA, Patrícia Valle de Albuquerque. Suicídio: a prática do psicólogo e os principais fatores de risco e de proteção. Universidade Federal 
Fluminense (UFF) de Rio das Ostras, 2015. Disponível: $<$ http://pepsic.bvsalud.org/pdf/igt/v12n23/v12n23a10.pdf $>$ Acesso em: 01/08/19

COUTO, Vilma Valéria Dias; TAVARES, Marcelo da Silva Araújo.Apegoe Risco de Suicídio em Adolescentes: Estudo de Revisão. Universidade do Distrito Federal, 2016. Disponível: <http://pepsic.bvsalud.org/pdf/rspagesp/v17n2/v17n2a10.pdf> Acesso em: $01 / 08 / 19$

JAHN, Guilherme Machado; DELL'AGLIO, Débora Dalbosco. A Religiosidade em Adolescentes Brasileiros. Universidade Federal de Psicologia, 2017. Disponível: $<$ http://pepsic.bvsalud.org/pdf/rpi/v9n1/04.pdf $>$ Acesso em: 01/08/19

KOLLER, Silvia; PAULA, Maria Clara de; HOHENDORFF, Jean Von ;Manual de Produção Científica. Porto Alegre. 2014.Disponível:<https://www.biosanas.com.br/uploads/outros/artigoscientificos/18/6505082 c2a7c23986651c7b1f7a4a92e.pdf> Acesso em: 01/08/19

MAGNANI,RafaelaMazoroski; STAUDT, Ana Cristina Pontello.Estilos Parentais e Suicídio na Adolescência: Uma Reflexão Acerca dos Fatores de Proteção. Centro Universitário FADERGS, 2018.2 Disponível em: $<$ http://pepsic.bvsalud.org/pdf/penf/v22n1/v22n1a07.pdf $>$ Acesso: 01/08/19

MOREIRA, Lenice Carrilho de Oliveira; BASTOS,Paulo Roberto Haidamus de Oliveira. Prevalência e fatores associados à ideação suicida na adolescência: revisão de literatura Universidade Federal de Mato Grosso do Sul - Campo GrandeMS, 2009. Disponível: $<$ http://www.scielo.br/pdf/pee/v19n3/2175-3539-pee-19-03-00445.pdf > Acesso em: 01/08/19

PATIAS, Naiana Dapieve, SILVA, Doralúcia Gil da; DELL'AGLIO,DéboraDalbosco. Exposição de adolescentes á violência em diferentes contextos: relações com a saúde mental.Universidade Federal do Rio Grande do Sul, Porto Alegre, RS, Brasil, 2016. Disponível em: $<$ http://pepsic.bvsalud.org/pdf/tp/v24n1/v24n1a10.pdf $>$ Acesso: 01/08/19 
SÁ, Daniel Graça Fatoride,et al.Fatores de Risco para Problemas de Saúde Mental na Infância/Adolescência. Universidade Presbiteriana Marckezine e Universidade de São Paulo, 2010. Disponível: < http://www.scielo.br/pdf/ptp/v26n4/08.pdf> Acesso em: 01/08/19

SCHLOSSER, Adriano;ROSA, Gabriel Fernandes Camargo; MORE, Carmen LeontinaOjedaOcampo. Revisão: Comportamento Suicida ao Longo do Ciclo Vital. $\begin{array}{lllll}\text { Universidade } & \text { Federal de } & \text { Santa } & \text { Catarina, } & \end{array}$ Disponível: $<$ http://pepsic.bvsalud.org/pdf/tp/v22n1/v22n1a11.pdf $>$ Acesso em: 01/08/19

SILVA, Liliane de Lourdes Teixeira, et al. O Suicidiona Adolescência nas publicações da enfermagem brasileira: Revisão Integrativa da literatura. Faculdade de Medicina da UFMG, 2015. Disponível em: $<$ http://www.seer.ufsj.edu.br/index.php/recom/article/view/767/939> Acesso: 01/08/19

VILHENA, Karime;PAULA, Cristiane Silvestre de. Problemas de conduta:Prevalência, Fatores de Risco/ Proteção; Impacto na vida escolar e adulta. Faculdade Prebisteriana de Marckezine, 2017. Disponível:<http://pepsic.bvsalud.org/pdf/cpdd/v17n1/v17n1a05.pdf $>$ Acesso em: 01/08/19

ZAPPE, Jana Gonçalves; DELL'AGLIO, Débora Dalbosco. Adolescência em diferentes contextos de desenvolvimento: risco e proteção em uma perspectiva $\begin{array}{lllll}\text { longitudinal.Universidade } & \text { Federal do } & \text { RS, }\end{array}$ Disponível: $<$ http://pepsic.bvsalud.org/pdf/psico/v47n2/02.pdf $>$ Acesso em: 01/08/19

ZAPPE, Jana Gonçalves; DAPPER,Fabiana. Drogadição na Adolescência: Família como Fator de Risco ou Proteção. Universidade de Passo Fundo (UPF), 2017.Disponível em: $<$ http://pepsic.bvsalud.org/pdf/rpi/v9n1/10.pdf > Acesso em: 01/08/19 